\title{
Poorly Developed Scenarios May Interface with Reliability of Triage Scales
}

\author{
(1) Amir Mirhaghi ${ }^{1}$, (1) Hojjat Shafaee ${ }^{2}$
}

${ }^{1}$ Nursing and Midwifery Care Research Center, Mashhad University of Medical Sciences, Mashhad, Iran

2Department of Disaster Public Health, Faculty of Public Health, Tehran University of Medical Science, Tehran, Iran

\section{Dear Editor,}

A higher level of agreement is expected for the patients in level 5 in the triage room that nurses would have fewer difficulties to prioritize them. The chief complaint of these patients is often easy to understand and uncomplicated. In this group, people have mild symptoms or injuries such as common cold or muscle strain. They are considered as non-urgent patients because they are not critically ill. These patients should bevisited within hours of their arrival by a physician. They form a significant proportion of the patients attending the emergency department. However, they do not have serious health problems and they may unnecessarily utilize available resources designated for urgent patients. They also may cause frustration in the workplace. Nurses believe that these patients interfere in the care of patients with serious illnesses (1).

Triage scales primarily aim to recognize emergency patients, so it is probable that they neglect to provide a precise definition for non-urgent ones. It is reported that level 5 of triage scales may not be defined as practically distinct from other levels (2). In this line, Ekins and Morphet (3) reported that consistency of triage decisions with the Australasian Triage Scale (ATS) level 5 is 0.47 , which is the lowest among other categories. They concluded that the overall level of inter-rater agreement is 0.4 or 'fair-to-good'. However, they attributed fair consistency to the poor judgment of emergency nurses, which may be due to the fact that the level 5 criteria are poorly developed within the ATS. Also, a recent study by Allen et al. (4) raised concerns about possible ambiguity in level 5 criteria in the ATS. They reported that interrater reliability is found to be kappa $=0.27$. In this study, pediatric nurses used the ATS criteria for triage 8 scenarios. Three of them are designated for level 5 . Nearly half of the patients were assigned to level 4 instead of level 5 in these three scenarios. This sort of disagreement may be due to the criteria overlap between level 4 and level 5 rather than low concordance among nurses.

The first scenario is defined as a 4 -year-old patient with $24 \mathrm{~h}$ sore throat, which isafebrile with no other symptoms. The second one is defined as a 3-year- old patient with earache overnight, now resolved. Temperature is $37.6^{\circ}$, respiratory rate is 16 , oxygen saturation is $98 \%$. The third one is defined as a 18 -month- old patient with struck in back of head by toy, having a $2 \mathrm{~cm}$ laceration with well approximated but not bleeding edges. There is no loss of consciousness. Patient is alert and active. It must be said that pain and level of consciousness are the most important criteria to influence nurses decisions in these scenarios. Nurses are allowed to assign alert children with mild pain to either level 4 or 5 using pediatric physiological discriminators. In fact, differences between level 4 and 5 criteria are limited to dehydration signs and symptoms and neurovascular compromise, so all three scenarios could be assigned to level 4 or 5 correctly. If scenarios were designated as representing distinct categories, reliability would be higher than what is reported by Allen et al. (4) While Craig et al. (5) have indicated that significant administrative concerns play a role in poor reliability among nurses in this study, we believe that the poorly developed scenarios for level 5 should be regarded as the key player. Researchers widely use scenarios to assess the reliability of triage decisions among nurses or physicians. It must be noted that scenarios must be strictly adjusted to the triage scales criteria in order to present 
concordance among nurses purely. Therefore, it is recommended level 5 criteria in ATS be revised.

Keywords: Reliability, triage, emergency, triage scales

\section{Ethics}

Peer-review: Externally peer-reviewed.

\section{Authorship Contributions}

Concept: A.M., H.S., Design: A.M., H.S., Data Collection or Processing: A.M., H.S., Analysis or Interpretation: A.M., H.S., Literature Search: A.M., H.S., Writing: A.M., H.S.

Conflict of Interest: No conflict of interest was declared by the authors.

Financial Disclosure: The authors declared that this study received no financial support.

\section{References}

1. Mirhaghi A, Heydari A, Ebrahimi M, Noghani Dokht Bahmani M. Nonemergent Patients in the Emergency Department: An Ethnographic Study. Trauma Mon. 2016;21:e23260.

2. Mirhaghi A, Ebrahimi M. Japan Triage and Acuity Scale needs to revise level 5 criteria. Emerg Med J. 2018;36:315.

3. Ekins K, Morphet J. The accuracy and consistency of rural, remote and outpost triage nurse decision making in one Western Australia Country Health Service Region. Australas Emerg Nurs J. 2015;18:227-33.

4. Allen AR, Spittal MJ, Nicolas C, Oakley E, Freed GL. Accuracy and interrater reliability of paediatric emergency department triage. Emerg Med Australas. 2015;27:447-52.

5. Craig SS, Cheek JA, Seith RW, West A, Goldie N. Accuracy and interrater reliability of paediatric emergency department triage. Emerg Med Australas. 2015;27:620-1 